\title{
ILCEA
}

Revue de l'Institut des langues et cultures

d'Europe, Amérique, Afrique, Asie et Australie

$11 \mid 2009$

Langues \& cultures de spécialité à l'épreuve des médias

\section{Restructuration du discours et image de Vladimir Poutine par Le Figaro}

\section{Valéry Kossov}

\section{OpenEdition}

Journals

Édition électronique

URL : http://journals.openedition.org/ilcea/127

DOI : 10.4000/ilcea.127

ISSN : 2101-0609

Éditeur

UGA Éditions/Université Grenoble Alpes

Édition imprimée

ISBN : 978-2-84310-179-3

ISSN : 1639-6073

Référence électronique

Valéry Kossov, «Restructuration du discours et image de Vladimir Poutine par Le Figaro », ILCEA [En

ligne], 11 | 2009, mis en ligne le 30 avril 2009, consulté le 08 mars 2021. URL : http://

journals.openedition.org/ilcea/127 ; DOI : https://doi.org/10.4000/ilcea.127

Ce document a été généré automatiquement le 8 mars 2021.

(C) ILCEA 


\title{
Restructuration du discours et image de Vladimir Poutine par Le Figaro
}

\author{
Valéry Kossov
}

1 Étant donné que c'est la publication du Figaro ${ }^{1}$ qui était à l'origine de ce travail d'analyse des versions multilingues de la conférence de presse tenue par Vladimir Poutine à Novo-Ogarevo à la veille du G8 en Allemagne en juin 2007 (voir texte de cadrage), nous commencerons par ce journal en mettant en relief certains éléments de comparaison tirés de la publication du journal russe Kommersant ${ }^{2}$, également présent à la conférence de presse.

\section{Mise en scène russo-française}

2 Alors que Le Figaro ne présente à ses lecteurs aucune mise en scène, se contentant de publier des questions et des réponses que nous analyseront plus tard, le quotidien russe Kommersant, représenté par le journaliste A. Kolesnikov, fait un compte rendu synthétique de la conférence sans reprendre ses quatre questions effectivement posées à V.Poutine. En revanche, le journal russe publie le procès-verbal intégral de la rencontre. D'une manière générale, le journaliste tente de rapprocher le lecteur au maximum de l'ambiance de Novo-Ogarevo en l'initiant à toute une série de détails sur les rapports entre les journalistes juste à la veille la rencontre. Notamment, Kommersant rapporte à ses lecteurs l'engagement pris par les journalistes présents de retarder la parution des articles relatant cette conférence du vendredi $1^{\mathrm{er}}$ juin, au lundi 4 juin. Cet engagement voulu par l'hebdomadaire Der Spiegel dont la version imprimée sort le lundi, n'a d'ailleurs pas été respecté par ce dernier, qui a publié les annonces circonstanciées de la rencontre dans sa version électronique dès le lendemain de la conférence. Sur un ton ironique qui lui a valu le qualificatif du « cabot très célèbre en Russie $»^{3}$, A. Kolesnikov évoque le mécontentement du journaliste italien qui tenait à 
faire sa publication dans l'immédiat et son soulagement face au désengagement du Spiegel.

3 Avec les mêmes intonations ironiques, A. Kolesnikov décrit les gestes des journalistes qui faisaient les signes d'un dernier adieu à leurs collaborateurs comme avant d'être envoyés au Goulag lorsqu'ils entraient dans la salle où devait se tenir la conférence.

4 Une partie importante de l'article russe est consacrée au repas au cours duquel s'est tenue la conférence de presse et au menu. L'ironie se sent même dans la description du président Poutine qui semble se désintéresser des questions récurrentes des journalistes et manifeste beaucoup plus d'enthousiasme pour son dessert, une soupe aux fraises de bois.

Enfin, la légèreté du ton produit un manque d'attention qui serait à l'origine du contresens dans la réexpression d'une réponse du président portant sur un sujet pourtant important. Notamment, d'après le procès-verbal, à la question du Times : «Êtes-vous d'accord avec le président Bush qui estime qu'il est inadmissible que l'Iran ait une arme nucléaire? » V. Poutine répond : « Oui, tout à fait d'accord ». Dans l'article de Kommersant la question est reformulée différemment : « Êtes-vous d'accord pour que l'Iran ait une arme nucléaire? » et la réponse est sauvegardée dans l'état : «Oui, tout à fait d'accord».

6 Enfin, le seul élément de la mise en scène qui n'ait pas été teinté d'ironie, c'est l'explication du retard du président, occupé à rendre visite à la veuve de B. Eltsine, à l'occasion des 40 jours après son décès.

7 Malgré certaines imprécisions et ce ton plutôt "bon enfant ", l'article russe, rédigé sous forme d'un compte rendu, ne suscite pas de remarques particulières concernant le contenu des questions et des réponses. Il donne une impression générale de l'ambiance et des thèmes abordés lors de cette conférence. Il n'en est pas de même dans le journal français qui publie les matériaux de la conférence sous forme d'interview avec V. Poutine. Cette forme place le lecteur dans un contexte plus concret du transfert des informations et nous pose des questions, d'une part sur la conformité à l'original et, d'autre part, sur l'impact de cette interview pour l'image de V. Poutine.

\section{Questions et thèmes abordés par Le Figaro}

8 Dès le début de la lecture comparative du procès-verbal de la conférence de presse et de l'entretien du Figaro, nous avons constaté une véritable reconstitution des questions et thèmes exposés. Cela nous amène, dans cette partie, à faire une brève présentation comparative des thèmes évoqués dans les questions effectivement posées lors de la rencontre par le journaliste du Figaro, d'après le procès-verbal, et des thèmes évoqués dans les questions et réponses publiées ensuite dans le journal. La version imprimée du Figaro présente l'interview comme « un entretien accordé par V. Poutine » au journal et indique le nom du journal devant la première question et le nom de V. Poutine devant la réponse en laissant ensuite des vides, ce qui laisse croire que les questions, comme les réponses, proviennent de la même source. Cela pourrait être considéré comme un faux malentendu entre le journal et le lecteur, car la première question proposée au lecteur du journal vient bien du Figaro. Ensuite, même si le nom du journal ne figure pas devant les questions, le lecteur reste bien dans l'idée qu'elles proviennent du 
journaliste français, ce qui est loin d'être le cas. Quelles sont donc les questions que Le Figaro a posées lors de la rencontre?

9 Parmi les quatre questions posées effectivement par Le Figaro, une seule a été reprise sous sa forme quasi intacte, bien qu'abrégée, ce qui s'explique probablement par le besoin d'économie d'espace sur les pages du journal. C'était pourtant une question presque protocolaire sur les futures relations entre le nouveau président français et son homologue russe. La question sur le statut du Kosovo a été posée par Le Figaro sous forme de réaction à la réponse donnée par V. Poutine au journaliste italien F. Venturini. Les deux autres questions étaient d'ordre économique et presque privé. La première, portant sur la possibilité des compagnies occidentales de participer à l'exploitation du gisement de gaz de Shtokman n'a pas été retranscrite dans l'interview. La deuxième, concernant les craintes que suscitent les sociétés russes en investissant dans le capital des entreprises européennes comme EADS, a été reproduite dans une version très abrégée.

10 Ainsi, nous pouvons constater qu'en réalité Le Figaro a posé quatre questions dont seulement trois se sont retrouvées dans le texte de «l'entretien du président russe ». D'où proviennent donc les 10 autres questions qui couvrent environ huit sujets politicoéconomiques différents?

11 Certes, nous apprenons de l'article russe que, en attendant l'arrivée du président, les journalistes se sont réparti les questions prioritaires à poser intéressant tous les Européens. Il est fort probable que Le Figaro n'a pas obtenu le droit de poser les questions sur les thèmes les plus pertinents du moment. S'est-il alors contenté de traduire et de reproduire les questions des autres et les réponses de V. Poutine?

12 Pour répondre à cette question, nous avons étudié en détail le procès-verbal et les interviews parus dans les journaux. Dans le cas du Figaro, il n'y a pratiquement pas de correspondances entre les formulations des questions et des réponses. Il n'est donc pas question ici de traduction, mais de la reconstitution des questions-réponses ou d'un exercice de synthèse qui s'apparente à une interview. La question qui se pose dans ce cas est de savoir à quel point cette reconstitution du discours est fidèle au procèsverbal en russe qui tiendra lieu, dans notre cas, de texte-source.

13 L'interview reconstituée du Figaro représente, certes, un travail de synthèse bien réfléchi et structuré. Les thèmes sont présentés en fonction de leur priorité et leur intérêt pour un lecteur français. En effet, l'interview commence par les rapports entre les chefs d'Etat qui sont implicitement assimilés aux relations entre les deux pays. Ensuite, vient le tour de l'actualité internationale de première importance (bouclier antimissile, G8) qui est suivi par l'actualité de « second plan » ('Iran, le Kosovo, l'affaire Lugovoii). Une fois les actualités politiques épuisées, le journal aborde des sujets économiques (pétrole, investissements russes). Enfin, l'interview se termine par un " fait divers » curieux et destiné à être drôle (Poutine - un démocrate pur) qui est ressenti comme "une phrase forte", un peu provocatrice, donnant lieu à des discussions, et par les futurs projets du président après l'expiration de son mandat.

Quels sont les procédés de cette reconstitution? D'abord, elle se fait par une reformulation abrégée qui ne tient pas compte du contexte général et conduit souvent à gommer certaines nuances de la question initialement posée. Ainsi, la question 4 de l'interview « Si Washington vous proposait de participer au bouclier antimissile, seriezvous prêt à en discuter avec l'OTAN ? » ne reflète pas tout à fait la teneur de la question 
originale du journal canadien The Globe and Mail, qui pourrait être résumée de la manière suivante : "Peut-être tout serait différent si l'OTAN participait davantage à ce projet du bouclier antimissile et si la Russie avait affaire à l'OTAN et non pas uniquement aux États-Unis. »

Cette suggestion d'approfondir des rapports avec un organisme transatlantique, qui serait mieux placé pour ce genre de projet de sécurité, n'est pas reproduite dans la version française, qui aurait tendance à identifier l'OTAN à Washington.

Un autre procédé que nous pouvons relever consiste à reformuler une question par fusion de deux questions sur le même sujet, sans pourtant reprendre le sens précis de ces deux questions. Il s'agit d'une question quasi inventée par le journaliste, afin de mieux placer sa mosaïque d'éléments de réponse.

La question« Que préconisez-vous pour le Kosovo dans la mesure où vous refusez l'indépendance proposée par le Plan Ahtisaari? » n'a été posée par personne sous cette forme. Elle est composée probablement par une partie de la question de l'italien $\mathrm{F}$. Venturini et de la réaction assez vive du journaliste français $\mathrm{P}$. Rousselin à la réponse de V. Poutine prônant le compromis dans la définition du statut du Kosovo.

F. Vebturini : « Je connais votre position par rapport au Kosovo et aux négociations directes entre les serbes et les kosovars. Ma question est, ne croyez-vous pas qu'une telle position contre le plan Ahtisaari à l'ONU motive réellement et pousse le Kosovo à la proclamation de l'indépendance unilatérale?»

P. Rousselin: «Je ne vois pas de possibilité de compromis. Donnez-moi une idée sur le compromis qui est possible ici. Soit le pays est indépendant, soit il ne l'est pas. Je ne comprends pas quel compromis est possible dans ce cas. Où voyez-vous la possibilité du compromis?»

Le sens de ces deux questions est proche. Cependant, tout le contexte émotionnel de la question en sort tronqué et une partie du sens se perd, notamment la notion du compromis (qui ne figure pas dans la réponse non plus).

Enfin, des omissions peuvent être relevées, même dans la première question protocolaire, qui évoque, dans sa version originale, à côté du nom de N. Sarkozy, celui de l'ex-président J. Chirac, ainsi que ses relations de travail avec V. Poutine. Dans la version de la question proposée au lecteur du Figaro, le nom de J. Chirac disparaît. Comparons :

Figaro (version originale) : M. le Président, vous allez rencontrer au sommet du G8 le nouveau président de la France, N. Sarkozy. Vous avez établi des rapports étroits de travail avec l'ex-président Chirac. Comment voyez-vous les rapports entre la Russie et la France pendant la présidence de N. Sarkozy que l'on considère comme un ami des États-Unis et qui, comme on le croit, serait concentré dans sa politique extérieure sur la question des droits de l'homme.

Figaro (version imprimée) : Vous allez faire la connaissance du président Sarkozy, présenté comme un ami des États-Unis et un défenseur des droits de l'homme. Comment voyez-vous l'évolution des rapports entre la Russie et la France?

Certes, les omissions et les versions très abrégées des questions peuvent être nécessitées par le souci d'économie d'espace du journal. Cependant, nous avons relevé également des ajouts, dont l'objectif est de compléter l'information sur un sujet probablement mal connu du public français. Cela pourrait être qualifié de compensation des lacunes d'informations et d'actualités présentées différemment dans les pays européens. Ainsi dans la question posée par le britannique Times, les journalistes français jugent opportun d'ajouter une partie, que nous avons mise en italiques, résumant brièvement l'affaire Lougovoï: "Certaines circonstances 
permettraient-elles d'extrader Andreï Lougovoï, inculpé par la justice britannique pour le meurtre d'Alexandre Litvinenko à Londres? » liées par une problématique commune. Examinons par exemple cette réponse reconstituée par Le Figaro:

Oui. 1.1 Nous sommes en train de revenir à cette époque. 1.2 Nos experts militaires nous disent que le système antimissile menace le territoire de la Russie jusqu'à l'Oural. 1.1 Si une partie du potentiel nucléaire des États-Unis est en Europe, nous devrons trouver une réponse. [Quelles sont ces mesures?] Bien sûr, nous devrons avoir des cibles en Europe. Quels moyens utiliserons-nous? Des missiles balistiques, des missiles de croisière ou de nouveaux systèmes d'armements, c'est une question technique. 1.3 Je suis contre toute course aux armements. Nous avons appris de l'expérience de l'URSS. Nous n'allons pas nous laisser entraîner. Les États-Unis vont 
dépenser des milliards et des milliards de dollars, nous allons bâtir une réponse asymétrique, beaucoup moins chère mais efficace.

\section{aussi déformer le
exemples suivants}

2.1 Nous sommes favorables (au dialogue) et à l'application de la loi internationale, qui prévoit le respect de l'intégrité territoriale des États. Si nous décidons de préférer à ce principe celui de l'autodétermination, il faut faire de même partout dans le monde, notamment en Ossétie du Sud, en Abkhazie et en Transnistrie. 2.2 À l'ouest, cette solution déclenchera des séparatismes en Europe. Voyez l'Écosse, la Catalogne, le Pays Basque... 2.1 Je ne pense pas que l'on doive humilier une nation européenne comme la Serbie en cherchant à la mettre à genoux. Il faut être patient, les possibilités de compromis n'ont pas été épuisées.

31 Une grande partie de cet exemple du Figaro (en 2.1) est une réponse à la question du Corriere della Sera avec une phrase (2.2) qui est tirée de la réponse "effective ", et par ailleurs assez développée, à la question du journal français. Cependant, le mot « dialogue » (entre parenthèses dans l'exemple) a été ajouté par les journalistes. Dans le procès-verbal de la conférence, V. Poutine insiste seulement sur l'application du droit international. Certes, nous pouvons supposer que le dialogue entre la Serbie et le Kosovo fait également partie des souhaits du président russe et que, globalement, le sens du propos est exacte.
Cependant, Le Figaro ajoute le même mot dans la réponse suivante qui ne concerne plus le conflit au Kosovo. Qui plus est, c'est toute une phrase qui a été ajoutée (3.1) : "Je suis absolument d'accord avec lui. Mais là encore nous sommes en faveur du dialogue. »

Il s'agit, cette fois, d'une réponse très brève et claire à la question du Times sur l'inadmissibilité de l'arme nucléaire pour l'Iran. Or la phrase ajoutée laisse supposer à 
un lecteur français que V. Poutine est favorable au dialogue sur la question de l'arme nucléaire pour l'Iran, ce qui déforme déjà sensiblement le sens de sa réponse sur ce sujet, négative sans ambiguïté.

Outre l'altération des nuances et du sens des propos recueillis lors de la reconstitution, se pose également le problème du style et de la syntaxe qui provient des coupures aléatoires dans le texte-source. En effet, la suppression de certaines parties de la phrase déforme les liens logiques du propos, ce qui crée un décalage stylistique par rapport au discours de Poutine en russe qui, formation juridique oblige, est généralement bien structuré et soumis à un ordre logique assez stricte avec souvent des tournures syntaxiques caractérisées par un grand nombre de phrases conditionnelles et impersonnelles. Ce type de discours, un peu procédurier en russe, se manifeste particulièrement dans les réponses aux questions à substrat juridique. Que devient ce discours dans la version du Figaro reprenant la question du Times relative à l'affaire Lougovoï?

Oui. Il faudrait modifier la Constitution russe. Mais les Britanniques n'ont pas substantiellement motivé leur requête d'extradition. Par ailleurs, une instruction est ouverte sur cette affaire en Russie. Si nos enquêteurs trouvent matière à inculper Lougovoï ou quiconque, il sera renvoyé devant les tribunaux. Si ceux qui ont transmis la demande d'extradition ignorent notre Constitution (qui empêche toute extradition, NDLR), leur compétence est en cause. S'ils connaissent notre Constitution, alors ce n'est qu'un geste politique. C'est de la stupidité ! Absurde! Les autorités britanniques ont laissé entrer sur leur territoire des voleurs et des terroristes. Elles sont responsables.

D'une part, nous pouvons constater des hiatus logiques (italique) ou un manque de liens entre les phrases exprimant des idées différentes, ce qui est motivé, peut-être, par un souci d'économie d'espace. L'original nous propose une réponse, certes, plus longue et plus développée mais mieux construite avec ses trois parties et une conclusion.

En revanche, Le Figaro accorde une large place à l'expression des émotions mettant deux points d'exclamation, ainsi que le mot "absurde », absent de l'original. Le mot "stupidité " a été effectivement employé mais dans une tournure beaucoup plus nuancée que celle de la reconstitution : «Quel que soit l'angle sous lequel on considère ce problème, tout cela n'est que stupidité. » (pas de point d'exclamation).

C'est d'ailleurs vers la fin de «l'entretien» que V. Poutine, dans la version du Figaro, commence à montrer davantage d'émotions, car les points d'exclamation se multiplient.

Avez-vous lu l'accord initial (de partage de la production) ? C'était un accord colonial, contraire aux intérêts de la Russie. Je regrette qu'au début des années 90 la Russie ait signé des accords pour lesquels les responsables auraient dû aller en prison! Cet accord ne rapporte rien, zéro! Mais les sociétés ont violé notre législation, c'est leur faute. Gazprom a reçu une proposition de s'allier à TNK-BP avant les problèmes environnementaux. Et quand ces problèmes sont survenus, Gazprom a sauvé ce projet en investissant 8 milliards de dollars.

Les hiatus logiques (en italique) auraient pu être évités si la réponse russe avait été bien traduite compte tenu du contexte. Le propos de l'original est nettement plus nuancé : «Les fonctionnaires russes se permettaient des choses pour lesquelles on aurait dû, en fait, les envoyer en prison. L'exécution de cet accord amenait à la situation dans laquelle la Russie laissait exploiter ses ressources naturelles sans rien obtenir en contrepartie. » Des transitions sont introduites entre ses phrases : « Mais si encore nos partenaires avaient respecté leurs engagements, on n'aurait eu aucune chance de 
changer cette situation. Toutefois ils deviennent, eux-mêmes, responsables après avoir violé la législation environnementale... »

Le passage relatif à la participation de Gazprom dans ce projet est introduit également par un mot de transition dans l'original, qui n'est pas reproduit dans la version du Figaro.

La réponse de V. Poutine se poursuit, avec des explications et des données chiffrées concernant le projet d'exploitation du gisement de Kovyktinskoe et se termine sur l'idée qu'il faut encourager les activités de BP en Russie. Tout cela est absent de la version du Figaro car jugé probablement peu intéressant pour ses lecteurs.

Dans les réponses aux dernières questions de "l'entretien", on constate que les phrases exclamatives se multiplient tandis que la syntaxe de V. Poutine se simplifie, avec des phrases plus courtes et un choix de tournures de moins en moins varié.

La question sur la crainte que provoque l'arrivée des investisseurs russes en Occident, est suivie d'une réponse assez émotionnelle exprimant une certaine indignation du président. La lecture de l'original ne traduit pas des sentiments d'indignation aussi forts. Bien que le sentiment d'étonnement de Poutine puisse être constaté (la traduction du même passage de l'original entre parenthèses), ses propos restent assez réservés et exprime une position qui n'est pas aussi agressive que dans la version reconstituée.

Pourquoi avoir peur? Les compagnies russes apportent l'investissement nécessaire en Europe. Elles ne volent pas! (De quoi ont-ils peur si les compagnies russes apportent des investissements qui sont extrêmement importants pour l'économie de tel ou tel pays européen ?... Ces compagnies viennent aux conditions du marché, elles ne viennent pas prendre quelque chose...) Elles créent des emplois. Si nous avions passé un accord d'investissement, des emplois auraient pu être sauvés chez Airbus, nous aurions évité des licenciements massifs. Nous sommes en train de créer notre holding d'aéronautique. Nous fabriquons par exemple le B200, un appareil de lutte contre les incendies. Pourquoi ne pas l'utiliser en Europe? Si l'Europe ne veut pas de nous, nous irons voir d'autres partenaires. Si Gazprom entre dans les réseaux de distribution européens, il sera intéressé [sic] de remplir les réseaux de gaz, où est le mal? On nous a critiqués à propos du gazoduc de la Baltique. On a dit qu'il court-circuitait des pays, mais nous ne fermons rien, c'est au contraire une route en plus.

Des raccourcis par rapport à l'original (en italique dans l'exemple français) sont à l'origine de nouvelles incohérences discursives.

le Poutine du Figaro hausse sensiblement le ton à la fin de son interview (en réalité, la « dernière » question a été posée par Der Spiegel au milieu de la conférence). En effet, dans la réponse, composée de sept phrases courtes, on a placé jusqu'à trois signes d'exclamation.

« Oui, bien sûr! Bien sûr que je suis un pur et absolu démocrate! La tragédie, c'est que je suis le seul pur démocrate au monde. Voyez les États-Unis : des tortures horribles, des sans-abri, Guantanamo. Voyez l'Europe: des manifestations violentes, durement réprimées. Même les Ukrainiens se sont discrédités et vont vers la tyrannie. Depuis la mort de Gandhi, je n'ai personne à qui parler !»

6 Certes, les journalistes ont le droit d'ajouter des signes d'exclamation aux phrases de la personne interviewée s'ils le jugent nécessaire pour transcrire l'oralité du discours. Cependant, cette version de réponse dans Le Figaro semble davantage agressive et provocatrice que la réponse de l'original. En effet, le procès-verbal nous rapporte le rire 
du président, ce qui signifie plutôt l'autodérision dans sa comparaison à Gandhi, même si, selon le journaliste russe A. Kolesnikov, cette ironie était bien dissimulée.

En revanche, Le Figaro omet les reproches assez justes mais provocateurs, que V. Poutine fait aux Occidentaux, d'utiliser contre les manifestants le gaz lacrymogène et des balles en caoutchouc.

\section{Conclusion}

Les procédés de reconstitution du discours analysés nous présentent un personnage froid, peu volubile, au langage relativement primitif, notamment dans le choix des tournures syntaxiques, mais qui se met facilement en colère et donc apparaît comme très menaçant. En somme, Le Figaro crée l'image d'un leader dictatorial, tiers-mondiste, assez agressif et peu instruit. Est-ce l'image qu'attend le lecteur français? Probablement oui, car elle est déjà constituée par les médias et surtout se réfère à des portraits de dictateurs connus $\mathrm{du} \mathrm{xx}^{\mathrm{e}}$ siècle. Dans ce cas, pourquoi ne pas renforcer cette image en adaptant ces propos des conférences de presse? Est-ce une image réelle et juste? Nous ne pouvons pas affirmer, à quelques rares exceptions, que les propos transcrits sont absolument faux. Cependant, une accumulation d'inexactitudes, de phrases mal traduites ou/et restituées hors contexte, des mots ajoutés et attribués à la personne, contribuent à constituer un personnage spécifique, décalé de la réalité, et créé sur mesure pour fâcher, indigner, provoquer, voire terroriser le public occidental. Est-ce vraiment l'objectif de V. Poutine de se faire une telle image? Il ne se fait probablement pas d'illusion quant à son image dans les médias occidentaux et son amélioration ne fait pas partie de ses priorités. Après tout ce n'est pas le public occidental qui vote aux élections russes et les favoris de l'Occident, comme M. Gorbatchev, ne sont pas automatiquement appréciés des Russes.

Ainsi, le souci de V. Poutine était plutôt de produire une bonne impression sur l'opinion publique russe à la veille des élections législatives fin 2007, d'inspirer au public russe ce sentiment de force tranquille qu'il a toujours incarné en Russie, de montrer qu'il lui est possible de se considérer vis-à-vis des Occidentaux comme un partenaire égal et d'exprimer ouvertement ses désaccords.

Dans cette logique, les objectifs de communication ont été réalisés aussi bien du côté des journalistes que de celui du président. Dans la mesure où les intérêts du pouvoir des médias et du pouvoir politique coöncident, le premier créant une image uniforme du second qui l'accepte et s'y adapte, force est de constater que le grand perdant dans cette situation est le lecteur. Celui-ci se fait une opinion à partir d'une réalité parfois artificielle et n'a pas le moyen de remettre en cause l'objectivité de l'image créée par la presse s'il n'a pas accès à un travail minutieux de comparaison multilingue. 


\section{NOTES}

1. Nodé-Langlois F., Rousselin P. «Poutine : "La Russie devra choisir des cibles en Europe" » Le Figaro 4.06.07

2. Колесников А. «Владимир Путин подал горячее. » Коммерсант 4.06.07.

3. Millot L. La Russie nouvelle. Actes Sud 2008, p. 196.

4. Les experts militaires occidentaux et russes estiment que pour changer de cibles il suffirait de reprogrammer l'ordinateur de commande de missiles, ce qui prendrait une dizaine de minutes. Donc cette menace de changer de cibles est utilisée tant par les journalistes que par V. Poutine pour impressionner le public.

\section{RÉSUMÉS}

Le présent article est consacré à la construction de l'image d'un homme politique par la restructuration de son discours dans la presse. En effet, dans notre société de l'image télévisuelle, le discours pourrait paraître un élément obsolète qui contribue peu à la renommée d'un politicien. Cependant, tant qu'existe la presse écrite, le discours politique ne saura perdre de son actualité. Ce qui semble poser un problème dans cette situation, c'est lorsque la presse prend la liberté de transférer le discours d'une personnalité en modifiant un certain nombre de ses aspects linguistiques, notamment, le style, la syntaxe, le choix d'expressions. Ces modifications transforment le contexte général des énoncés et, par conséquent, l'objectif de la communication. Ce genre de transformation est étudié d'après l'exemple de la publication dans Le Figaro de la conférence de presse de l'ex-président russe V. Poutine devant les journalistes des pays membres du G8.

This paper studies the building of a politician's image through the restructuration of his discourse in the press. In our television dominated society, discourse might seem an obsolete element contributing little to a politician's fame. Yet, as long as print press remains in circulation, political discourse will keep its relevance. What seems to generate a problem in this situation is the liberties taken by the press when transferring the discourse of a given personality through linguistic modifications relative to syntax, stylistics and choice of expressions. These modifications transform the contextual embedding of the statements and, as a consequence, the purpose of the communication. This paper highlights such transformation procedures through an analysis of the article published by Le Figaro following the press conference given by Vladimir Putin, Russia's ex-President, to a panel of journalists from G8 countries.

\section{INDEX}

Mots-clés : conférence de presse, discours politique, presse écrite, Poutine Vladimir, G8, image Keywords : press conference, political discourse, print press, Putin Vladimir, G8, image 
AUTEUR

VALÉRY KOSSOV

ILCEA / GREMUTS 